\title{
The Calculated Circular Dichroism of Polyproline II in the Polarizability Approximation
}

\author{
E. W. RONISH and S. KRIMM, Harrison M. Randall Laboratory of \\ Physics, University of Michigan, Ann Arbor, Michigan 48104
}

\begin{abstract}
Synopsis
The circular dichroism (CD) spectrum of polyproline II (PPII) has heretofore been moderately well calculated from exciton theory only at the expense of assuming unreasonable chain conformations and accepting a conservative spectrum in the 180250-nm region (which is not observed). We have incorporated far uv transitions in the polarizability approximation and, together with the $\pi_{2} \pi^{*}$ transition, have calculated the resulting correction to the exciton model. This has been accompanied by a modified assignment of the $\pi \pi^{*}$ transition in PPII, and a simultaneous calculation of the absorption and CD spectra of the $\alpha$-helix, $\beta$ structure, PPI, and PPII. We obtain good agreement with the observed CD spectrum of PPII in the 180-250-nm region for acceptable chain conformations. In addition, we predict a negative $\mathrm{CD}$ into the far uv, in agreement with recent experimental observations. Our calculations also reproduce features of the far uv CD spectrum of the $\alpha$-helix, and are in agreement with the CD spectra of the $\beta$ chain and PPI. The calculated CD of the unordered polypeptide chain is not significantly influenced by far uv contributions, indicating that our previous calculation is valid for such a system. These results demonstrate the importance of incorporating far uv transitions in order to achieve an adequate theoretical explanation of the CD spectra of polypeptides.
\end{abstract}

\section{INTRODUCTION}

The calculation of the circular dichroism (CD) spectrum of poly-Lproline II (PPII) has been the subject of a number of studies. ${ }^{1-4}$ Despite the relatively satisfactory agreement obtained for other polypeptide chain conformations by means of such calculations, ${ }^{2,3,5-10}$ the CD spectrum of PPII has not been accounted for satisfactorily. This is the case from at least three viewpoints. 1) In order to get any thing approaching reasonable agreement, conformations have had to be assumed ${ }^{3,4}$ that are not consistent with the results of conformational energy calculations. ${ }^{11-15}$ 2) The assignment of the $\pi \pi^{*}$ transition to the observed strong negative $C D$ band near $206 \mathrm{~nm}$ is not consistent with the observed parallel polarization of this band in linear dichroism studies. ${ }^{16,17}$ 3) While all calculations done thus far predict a conservative CD spectrum between 180 and $250 \mathrm{~nm}$, the observed CD of PPII in this region is basically nonconservative. ${ }^{18,19}$ It is therefore appropriate to reexamine the calculation of the CD spectrum of PPII, and in particular the assumptions that have been made heretofore.

(C) 1974 by John Wiley \& Sons, Inc. 
In this paper we have recalculated the CD spectrum of PPII on the basis of a reassignment of the $\pi \pi^{*}$ transition and of the inclusion of far uv transitions, in part in the polarizability approximation. We obtain very satisfactory agreement with the CD spectrum of PPII in the 180$250-\mathrm{nm}$ region, using acceptable chain conformations. The spectra of other polypeptide chain conformations are also well reproduced. In the region below $180 \mathrm{~nm}$ the PPII spectrum is predicted to be nonconservative until the region of the far uv transitions. This prediction differs significantly from that of previous calculations, and thus provides for the possibility of a clear experimental test of the theory.

\section{ASSIGNMENT OF $\pi \pi^{*}$ TRANSITION ENERGY}

As we have noted, in previous calculations the $\pi \pi^{*}$ transition has been associated with the intense negative CD band near $206 \mathrm{~nm}$. There are several reasons for questioning this assignment and reassigning this $\mathrm{CD}$ band to one component of a split transition. First, a study of the uv absorption spectra of oligomers of proline ${ }^{20}$ suggests that the absorption near $206 \mathrm{~nm}$ in PPII (and PPI) is an enhanced component of a two-band spectrum, the other component being at 180-185 $\mathrm{nm}$. We reproduce in Figure 1 the absorption spectra of the proline dimer and tetramer as well as that of PPII, and compare these to the spectrum of the $\alpha$-helix. The proline monomer absorbs near $182 \mathrm{~nm},{ }^{20}$ and we can see that with increased degree of polymerization the center of the transition shifts (to about 198 $\mathrm{nm}$ in PP) and a splitting occurs (with components near 185 and $206 \mathrm{~nm}$ in PP). A similar result is noted for the $\alpha$-helix, but whereas in the $\alpha$ helix the short-wavelength component is enhanced, in PP the intensity of the long-wavelength component becomes strongest in the polymer. Second, a study of the lincar dichroism of the $\alpha$-helix ${ }^{21}$ has shown that the 208-nm band has parallel polarization. As we have noted, such studies on PPII ${ }^{16,17}$ show that its strong 206-nm band has parallel polarization, supporting our contention that it is onc component of a split transition. Finally, calculations that use an exciton formalism ${ }^{22}$ indicate that the long-wavelength band should exhibit parallel polarization, just as is expected of the $\alpha$-helix. ${ }^{23}$

We therefore conclude that the $\pi \pi^{*}$ transition of PPII is essentially similar to that of other polypeptides in having two major bands. We estimate that the shorter wavelength component is observed at $189 \mathrm{~nm}$ in the $\alpha$-helix and at $185 \mathrm{~nm}$ for PP, and the longer wavelength component at $204 \mathrm{~nm}$ for the $\alpha$-helix and at $206 \mathrm{~nm}$ for PP. The calculations, of course, must then account not only for the CD spectra of PPI and PPII, but also for the intensity enhancement in this case of the long wavelength band as well as the shift in the center of the transition from the monomer to the polymer.

\section{FAR ULTRAVIOLET TRANSITIONS}

In the exciton formalism the splitting of the $\pi \pi^{*}$ polymer transition comes about naturally from the interaction between such transitions in 


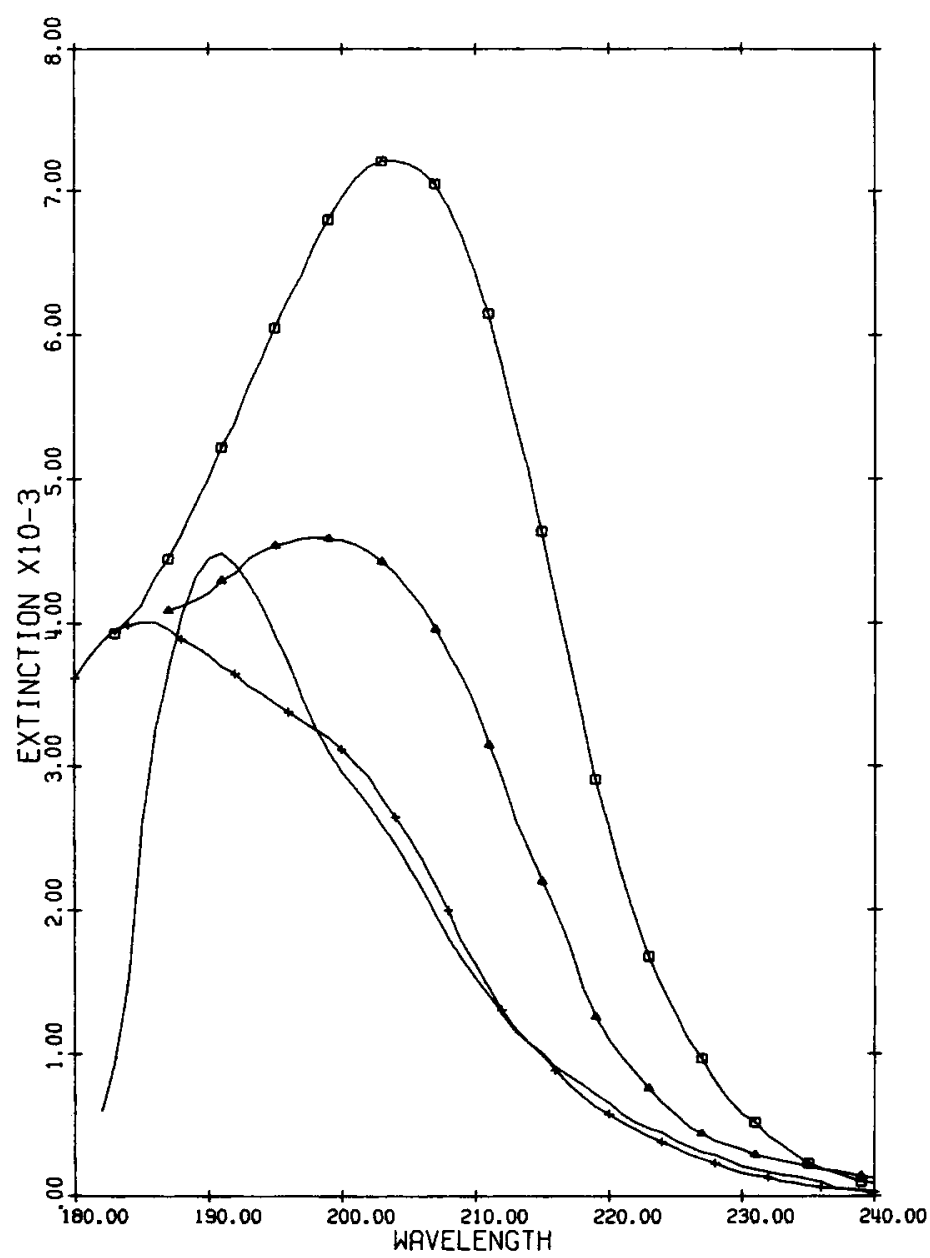

Fig. 1. Ultraviolet absorption spectra of proline dimer $(+)$, proline tetramer $(\Delta)$, and polyproline II ( $\square$ ), from Ref. 20, and of the $\alpha$-helix (solid line), from Ref. 21.

neighboring monomer units. This interaction cannot, however, lead to a shift in the center of the transition in proceeding from monomer to polymer. Such a shift can only arise from a "field effect" which other transitions in adjacent monomers exert on the $\pi \pi^{*}$ transition in a given monomer. We believe that these are the transitions of the peptide group in the far uv. Their perturbation of the $\pi \pi^{*}$ transition would be responsible, for example, for the shift of the transition from $182 \mathrm{~nm}$ in the proline monomer to about $198 \mathrm{~nm}$ in the polymer. Similarly, intensity changes in the absorption spectrum of the polymer relative to the monomer [hyperchromism in $\mathrm{PP},{ }^{20}$ hypochromism in the $\alpha$-helix ${ }^{24}$ ] are attributable to a perturbation of the $\pi \pi^{*}$ transition by the far uv transitions.

In the absence of complete information on the far uv transitions of the peptide group, their effect can be approximated by a polarizability correction..$^{5,25}$ We have chosen to utilize this approach, and have calculated 
the perturbation of both the $\pi \pi^{*}$ and the $n \pi^{*}$ transitions by the far uv transitions in the polarizability approximation. It should be noted that the claim ${ }^{3}$ that far uv transitions are not important in accounting for the CD spectra of polypeptides is not supported by our calculations. The reason for this discrepancy in results is that the above authors ${ }^{3}$ included one general transition placed artificially at $160 \mathrm{~nm}$ in order to test this point. They interacted this with the $\pi \pi^{*}$ transition and found that no improved CD spectrum of PPII was calculated. This is not surprising, since a proper model should include many transitions: an appropriate asymmetric environment for the $\pi \pi^{*}$ transition is not created by one far uv transition alone. A better approximation to the true asymmetric environment would be expected by incorporating all transitions, and it has been shown ${ }^{25}$ how this can be achieved with polarizability ellipsoids. As we shall show, a significant improvement is possible by this approach.

The equations and polarizability ellipsoids used in the present work are given in the Appendix and Table I, and are essentially identical to those used previously. ${ }^{5}$ Although $n^{\prime} \pi^{*}$ and $n \sigma^{*}$ transitions had previously been incorporated explicitly, ${ }^{5}$ we have not done so. This is because they have not generally been agreed upon, their inclusion as previously parametrized ${ }^{5}$ leads to disagreement with the PPII spectrum, and experimental studies ${ }^{26}$ rule out their assignment to observed bands because these have the wrong polarization. We have, however, included the $\pi_{2} \pi^{*}$ transition, placing it at $133 \mathrm{~nm}$. Even though this transition contributes very little to the calculated spectra, except for the $\alpha$-helix, we have included it because its assignment is less uncertain. ${ }^{26}$ All of the other far uv transitions are therefore incorporated into onc transition, placed at $100 \mathrm{~nm}$, and their effect on the spectrum is calculated by the polarizability approximation. It should be noted that, while the latter permits a calculation of the contribution to the rotational strengths, it does not provide the position at which this contribution is to be placed. Following earlier practice, ${ }^{6}$ we have placed the $\pi \pi^{*}$ polarizability contribution at the location of the parallel component of the $\pi \pi^{*}$ uv absorption transition, the $n \pi^{*}$ polarizability contribution being placed similarly at the position of the $n \pi^{*}$ transition.

\section{CHOICE OF MONOPOLES}

In order to determine whether the results are sensitive to the choice of monopoles, calculations were done with three sets: set I were the Schellman monopoles, ${ }^{27}$ set II were those previously used by us, ${ }^{10}$ and set III were the Woody-Tinoco monopoles ${ }^{5}$ modified by semiempirical screening coefficients and a split ground state. ${ }^{6}$ The major features of the calculations were independent of the monopoles used, but some features were sensitive to the choice made.

All sets of monopoles give the same exciton $\pi \pi^{*}$ rotational strengths. However, they all underestimate the observed splittings between the parallel and perpendicular bands in the uv spectra. We found that if we calculated the interaction matrix element between $\pi \pi^{*}$ transitions in a dipole- 
TABLE I

Parameters of the Calculation

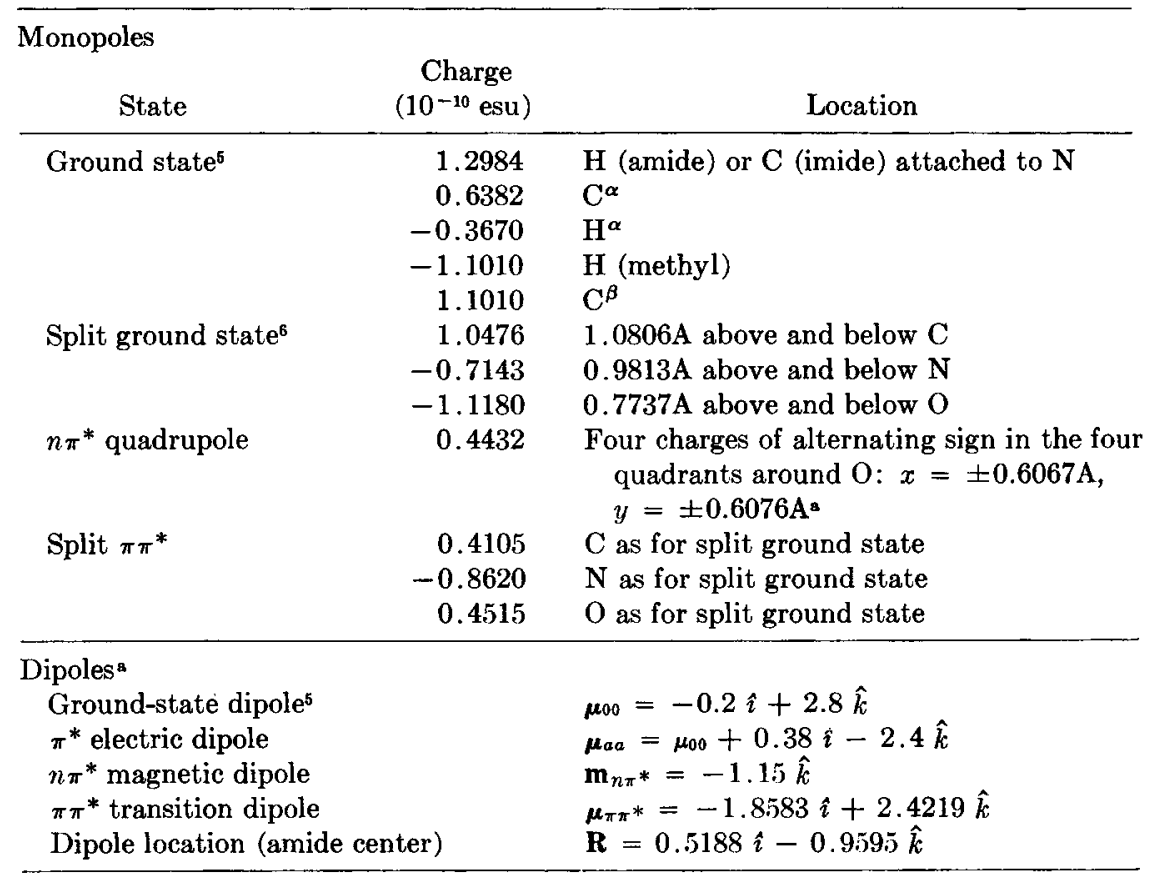

Polarizable Group Parameters

\begin{tabular}{lllc} 
& Direction of Principal & \multicolumn{2}{c}{ Magnitude $\left(\mathrm{A}^{3}\right)$} \\
Location & \multicolumn{1}{c}{ Axis } & $\alpha_{11}$ & $\alpha_{33}-\alpha_{11}$ \\
\hline Amide center & $\perp$ amide plane & 3.79 & -0.76 \\
Bond center & $\mathrm{N}-\mathrm{C}^{\alpha}$ & 0.633 & -0.033 \\
Bond center & $\mathrm{C}^{\alpha}-\mathrm{C}^{\prime}$ & 0.271 & 0.73 \\
Bond center & $\mathrm{C}^{\alpha}-\mathrm{H}^{\alpha}$ & 0.64 & 0 \\
Bond center & $\mathrm{C}^{\alpha}-\mathrm{C}^{\beta}$ & 0.271 & 0.73 \\
Group center & $\mathrm{C}^{\alpha}-\mathrm{C}^{\beta}\left(\mathrm{CH}_{3}\right.$ axis $)$ & 1.92 & 0 \\
\hline
\end{tabular}

\begin{tabular}{ccc}
\hline $\begin{array}{c}\text { Transition Energies } \\
\text { Transition }\end{array}$ & Amide $(\mathrm{nm})$ & Imide (nm) \\
\hline$\pi \pi^{*}$ & 190 & 198 \\
$n \pi^{*}$ & $220(\alpha)$ & 230 \\
& $215(\beta)$ & - \\
Pol'y & 100 & 100 \\
\hline
\end{tabular}

Conformations and Bandwidths

\begin{tabular}{lrrrcc} 
& & & \multicolumn{3}{c}{ Bandwidths (nm) } \\
Structure & $\omega$ & $\varphi$ & $\psi$ & Exciton & Pol- $R_{\pi \pi^{*}}$ \\
\hline$\alpha$ & 180 & -47 & -62 & 12 & 11 \\
$\beta$ & 180 & -140 & 140 & 10 & 10 \\
PPI & 0 & -80 & 170 & 30 & 10 \\
PPII & 180 & -65 & 165 & 14 & 10 \\
\hline
\end{tabular}

a Coordinate system with origin at $\mathrm{O}$ atom, $z$ axis along $\mathrm{C}^{\prime} \rightarrow \mathrm{O}$ direction, $x$ axis in $\mathrm{N}-\mathrm{C}^{\prime}-\mathrm{O}$ plane toward $\mathrm{N}-\mathrm{C}^{\prime}$ direction, $y$ axis perpendicular to $\mathrm{N}-\mathrm{C}^{\prime}-\mathrm{O}$ plane and forming a right-handed coordinate system. 
dipole rather than a monopole-monopole approximation, the calculated splittings were larger and generally in better agreement with experiment. Reasonable exciton splittings in PPII have previously been obtained using a dipole-dipole approximation. ${ }^{22}$ This is not a major point, however, since all sets are qualitatively the same in calculating $\pi \pi^{*}$ rotational strength splittings via an exciton secular determinant.

The $n \pi^{*}$ rotational strengths, on the other hand, are very sensitive to certain specific features of the monopoles. (The calculation usually gives rotational strengths that cluster within a few nanometers of the parametrized $n \pi^{*}$ energy, thus making it possible to speak meaningfully of the $n \pi *$ rotational strength, which we symbolize by $R_{n \pi *}$.) We find that the exciton (exc)- $R_{n \pi}$ is affected slightly by the positions of the $n \pi^{*}$ monopoles, i.e., whether they are close to the oxygen atom or far away, and also by the positions of the amide ground-state monopoles. The most important factor affecting exc- $R_{n \pi^{*}}$, however, is the magnitude of the magnetic dipole moment $\mathbf{m}_{n \pi *}$ of the $n \pi^{*}$ transition. We find that exc- $R_{n \pi *}$ is directly proportional to $\mathbf{m}_{n \pi *}$. Since various values have been taken for $\mathbf{m}_{n \pi}$ (varying from $0.58 \mathrm{BM}^{5}$ to $0.9 \mathrm{BM}^{6}$ to $1.3 \mathrm{BM}^{27}$ ), and since this quantity is not known experimentally, we have taken $\mathbf{m}_{n \pi^{*}}$ as a parameter. The best results are obtained with $\mathbf{m}_{n \pi}=1.15 \mathrm{BM}$, which is a reasonable value.

The polarizability contribution to the rotational strengths is found to be independent of the monopoles for the $\pi \pi^{*}$ transition. Thus, the characteristic polarizability contribution due to interaction with the $\pi \pi^{*}$ transition, which we find to be large and negative for the $\alpha$-helix and PPII and small for the $\beta$ structure and PPI, is independent of the parametrization although it is sensitive to the conformation. This is not the case for the polarizability contribution to the rotational strengths due to the $n \pi^{*}$ transition, pol- $R_{n \pi^{*}}$. We find that pol- $R_{n \pi^{*}}$ is directly proportional to $\mathbf{m}_{n \pi^{*}}$, and is small for every conformation except the $\alpha$-helix. For the $\alpha$ helix, pol- $R_{n \pi^{*}}$ is positive and larger than exc- $R_{n *}$ (which is of course negative) for our previous, ${ }^{10}$ and for Bayley et al's, ${ }^{27}$ monopoles. Thus, these two sets of monopoles would give a net positive $n \pi$ * rotational strength for the $\alpha$-helix, contrary to experimental observation. For the set of monopoles reported in this paper, which differs only in that it has no $n \pi^{*}$ monopoles around the carbon atom, pol- $R_{n \pi^{*}}$ is positive but small. The result is that the net $n \pi^{*}$ rotational strength in the $\alpha$-helix is negative and proportional to $\mathbf{m}_{n \pi *}$. Thus, the inclusion of the far uv transitions by means of the polarizability approximation requires us to calculate a small value of pol- $R_{n_{\pi} *}$ in order to be in agreement with experiment. This in turn seems to suggest that the nonbonding $n$ orbital does not spread out, as assumed by some authors, ${ }^{27}$ but is localized on the oxygen atom, as proposed earlier. ${ }^{5}$

An interesting feature of the results, which is independent of the choice of monopoles, concerns the CD spectrum of PPII. In this case pol- $R_{n \pi *}$ is negative and small, and exc- $R_{n \pi}$ is positive and small, but the net $n \pi^{*}$ rota- 
tional strength is always negative. Thus, the weak positive $\mathrm{CD}$ band observed near $218 \mathrm{~nm}^{18,19}$ is not to be associated with an $n \pi^{*}$ transition, and this conclusion is independent of the parametrization. As we shall see later, it probably arises from an overlap of the nearby component of the $\pi \pi^{*}$ transition.

\section{RESULTS AND DISCUSSION}

The calculation of the exciton $\pi \pi^{*}$ and $n \pi^{*}$ rotational strengths followed the methods used previously. ${ }^{10}$ The calculation of the polarizability contributions was based on the equations derived by Tinoco ${ }^{25}$ and Woody and Tinoco. ${ }^{5}$ We give in the Appendix the equations that we used to obtain the polarizability corrections to the energy, the dipole strength, and the rotational strength.

Calculations were done on the shift in position of the $\pi \pi^{*}$ and $n \pi^{*}$ transitions in going from the monomer to the polymer. The results, although qualitatively correct (the center of the transition shifts to longer wavelengths with increasing chain length), overestimated the shift quantitatively, in some cases by a factor of 2-3. We have therefore not depended on such calculations, but have taken the center of the transition as a parameter. The values chosen for the amide were $\pi \pi^{*}-190 \mathrm{~nm}$ and $n \pi^{*}-220 \mathrm{~nm}$ ( $\alpha$-helix) and $215 \mathrm{~nm}$ ( $\beta$ structure); for the imide these were $\pi \pi^{*}-198 \mathrm{~nm}$ and $n \pi^{*}-230 \mathrm{~nm}$. These values are consistent with those found in model compounds. ${ }^{28}$ It should be noted that the PPI and PPII $\pi \pi^{*}$ transitions are not centered near $206 \mathrm{~nm}$, which we have shown should be assigned to the parallel component of the transition in uv absorption.

These, as well as other, parameters of the calculation are given in Table I. The centers of the transitions as well as the bandwidths apply to both the absorption and the CD calculations. It will be seen that the exciton bandwidths are similar, about $12 \mathrm{~nm}$, except for PPII, where they are 30 $\mathrm{nm}$. This is not unreasonable, since the PPII structure is somewhat more flexible about its minimum than is true for the other conformations. ${ }^{15}$ The polarizability bandwidths are all near $10 \mathrm{~nm}$, which may be justified in that the "field effect" of the far uv transitions might be expected to be less sensitive to conformational flexibility.

The calculations for the $\alpha$-helix, $\beta$ structure, and PPI and PPII structures were all done for chains 20 peptide units long, assuming an alanyl (i.e., methyl) side-chain residue.

\section{Absorption Spectra}

The calculated absorption spectra of the $\alpha$-helix, $\beta$ chain, and PPI and PPII structures are shown in Figures 2-5, together with the observed polymer spectra. The calculations include a contribution, called $H_{1}$, which represents the polarizability interaction with the $\pi \pi^{*}$ transition (see Appendix). This contribution, which is positive for the $\beta$, PPI, and PPII structures and negative for the $\alpha$-helix, is given in Table II. When added 


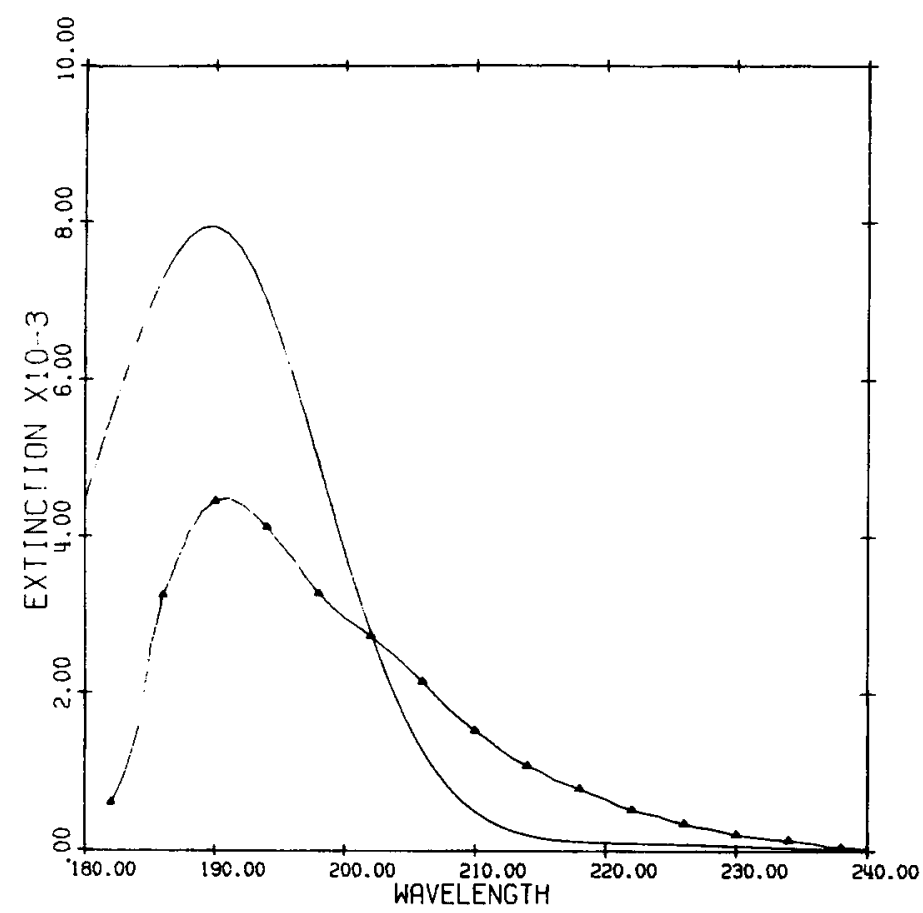

Fig. 2. Calculated $(\longrightarrow$ ) and observed ( $\Delta$ ) absorption spectra of the $\alpha$-helix.

to the dipole strength associated with the exciton contribution $\left(3.05^{2}=\right.$ $9.303 \mathrm{D}^{2}$ ), we obtain the predicted integrated absorption.

It can be seen from Table II that the observed hypochromism of the $\alpha$ helix relative to the monomer is predicted by the calculation, although the large magnitude $(4.8-9.3=-4.50)$ is not well reproduced. The small hypochromism of the $\beta$ structure is not predicted, a hyperchromism being in fact calculated. This may be due to the fact that the calculation was done for a single chain instead of the more realistic antiparallel chain pleated sheet structure. The spectra of PPI and PPII are reasonably well reproduced. Thus, important qualitative and some quantitative features of the observed absorption spectra can be accounted for by the incorporation of a polarizability contribution for the far uv transitions.

\section{Circular Dichroism Spectra}

The calculated CD spectra of some regular structures are given in Figures 6-9. Detailed features of these, and the spectra of unordered structures, are considered below.

$\alpha$-Helix. The CD spectrum of the $\alpha$-helix is given in Figure 6. Some of the calculated rotational strengths are given in Table II, and compared to observed values. It will be seen from Figure 6 that the calculation gives good agreement with the observed CD spectrum in the 180-250-nm region, and that it reproduces quite reasonably some of the main features recently 


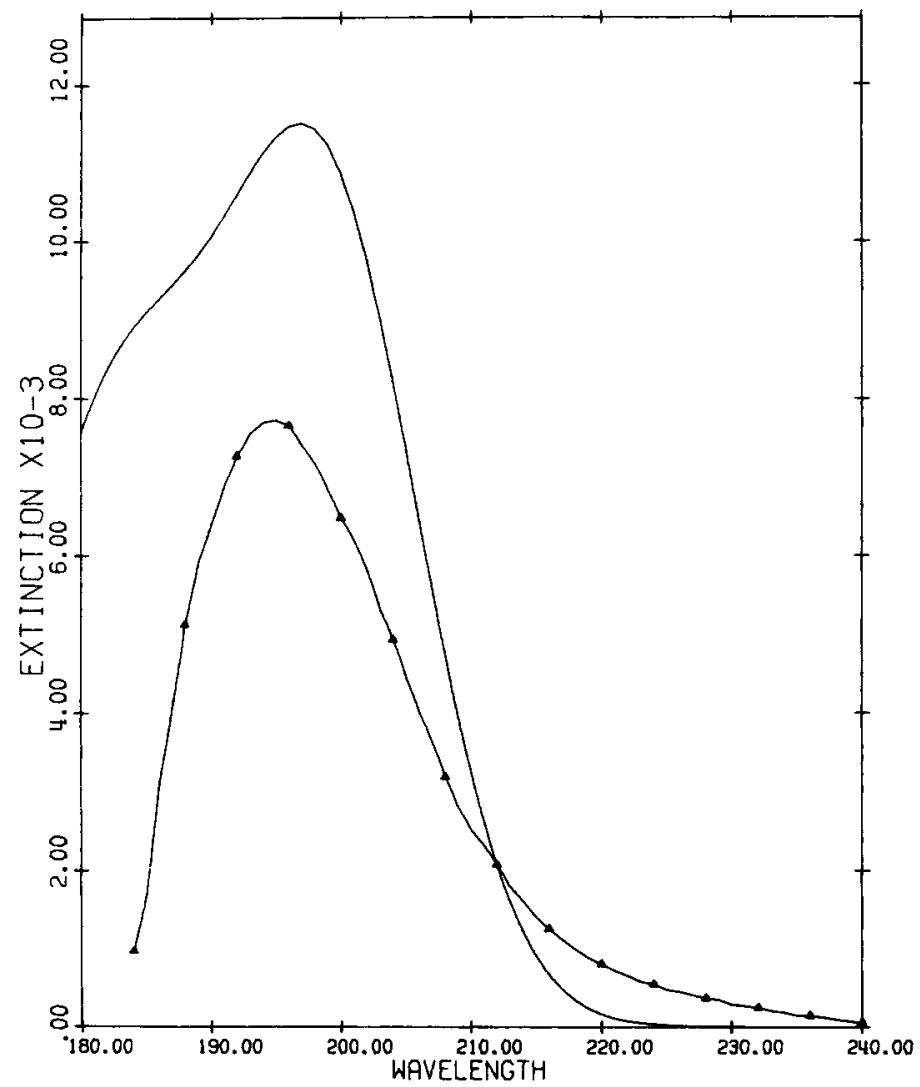

Fig. 3. Calculated (- - ) and observed ( $\Delta$ ) absorption spectra of the $\beta$ structure.

TABLE II

Calculated and Observed Absorption and CD spectra

\begin{tabular}{|c|c|c|c|c|c|}
\hline \multicolumn{6}{|c|}{ Absorption Spectra } \\
\hline Structure & Exciton $\left(\mathrm{D}^{2}\right)$ & \multicolumn{2}{|c|}{$H_{1}\left(\mathrm{D}^{2}\right)$} & $\epsilon$ (calc) & $\epsilon$ (obs) \\
\hline$\alpha$ & 9.303 & \multicolumn{2}{|c|}{-1.429} & 7.874 & 4.80 \\
\hline$\beta$ & 9.303 & & 6.812 & 16.115 & 8.00 \\
\hline PPI & 9.303 & \multicolumn{2}{|r|}{4.822} & 14.125 & 12.80 \\
\hline PPII & 9.303 & & 2.888 & 12.191 & 13.40 \\
\hline \multicolumn{6}{|l|}{ CD Spectra } \\
\hline & \multicolumn{2}{|c|}{ Pol- $R_{\pi \pi *}(\mathrm{dBm})$} & & \multicolumn{2}{|c|}{$\Sigma n \pi^{* b}$} \\
\hline Structure & Calc & $\mathrm{Obs}^{\mathrm{a}}$ & Pol- $R_{n \pi} *$ (calc) & Calc & Obs \\
\hline$\alpha$ & -0.499 & -0.3 & 0.108 & -0.268 & -0.23 \\
\hline$\beta$ & 0.049 & 0 & -0.024 & -0.123 & — \\
\hline PPI & -0.391 & 0.2 & 0.011 & -0.060 & $\ldots$ \\
\hline PPII & -0.416 & -0.5 & -0.102 & -0.069 & $-c$ \\
\hline
\end{tabular}

a $\left[R_{\pi \pi^{*}}(\mathrm{obs})\right]-\left[\mathrm{exc}-R_{\pi \pi^{*}}(\mathrm{calc})\right]$.

${ }^{b}\left[\operatorname{exc}-R_{n \pi *}\right]+\left[\mathrm{pol}-R_{n \pi} *\right]+$ vibronic contribution. ${ }^{30}$

- Not readily separable from observed CD spectrum. 


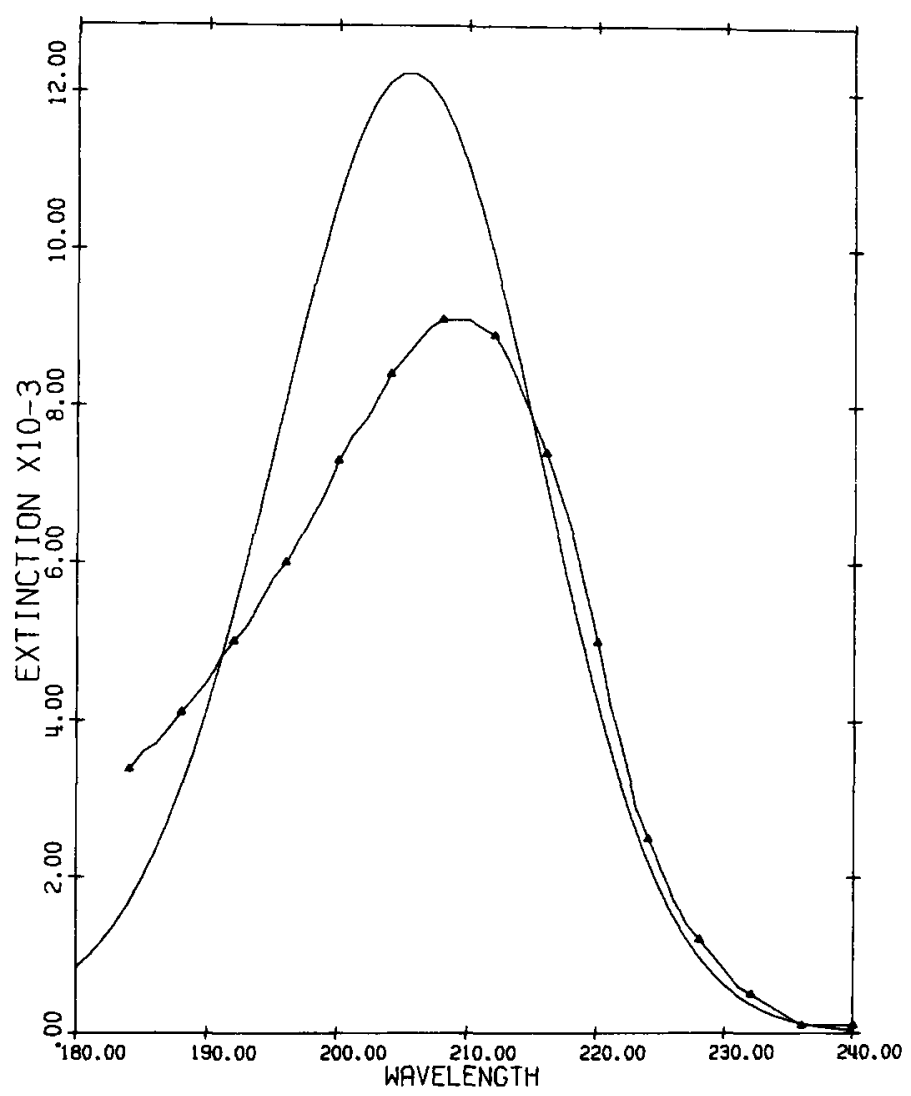

Fig. 4. Calculated (-) and observed $(\Delta)$ absorption spectra of polyproline I.

observed in the far uv spectrum. ${ }^{29}$ This includes a negative band near 170 $\mathrm{nm}$ and a band pair near $135 \mathrm{~nm}$. The details at shorter wavelengths are, of course, not expected to be reproduced in detail since the far uv transitions have been lumped together into one contribution placed at $100 \mathrm{~nm}$. It should be noted that the bands near $135 \mathrm{~nm}$ arise explicitly from the $\pi_{2} \pi^{*}$ contribution. They are dominant for the $\alpha$-helix conformation, but do not show up so specifically for other conformations. The $170-\mathrm{nm}$ band is predicted to be less intense for longer chains, which may in part account for the poor agreement when compared to our calculation for a $20-\mathrm{mer}$. There may also be other transitions contributing in this region, which we have. neglected.

As can be seen from Table II, pol- $R_{\pi \pi *}$ is calculated in fair agreement with the observed value, if we can assume the latter to be given by the observed component of the $\pi \pi^{*}$ transition (near $208 \mathrm{~nm}$ ) from which is subtracted the calculated exciton contribution. The calculated $n \pi^{*}$ ellipticity is in fair agreement with that observed. It will be scen that pol- $R_{n \pi^{*}}$ represents about $40 \%$ of the total $n \pi^{*}$ ellipticity. (The exc- $R_{n \pi *}$ rotational strength includes a contribution from vibronic coupling with the $\pi \pi^{*}$ transition, ${ }^{30}$ 


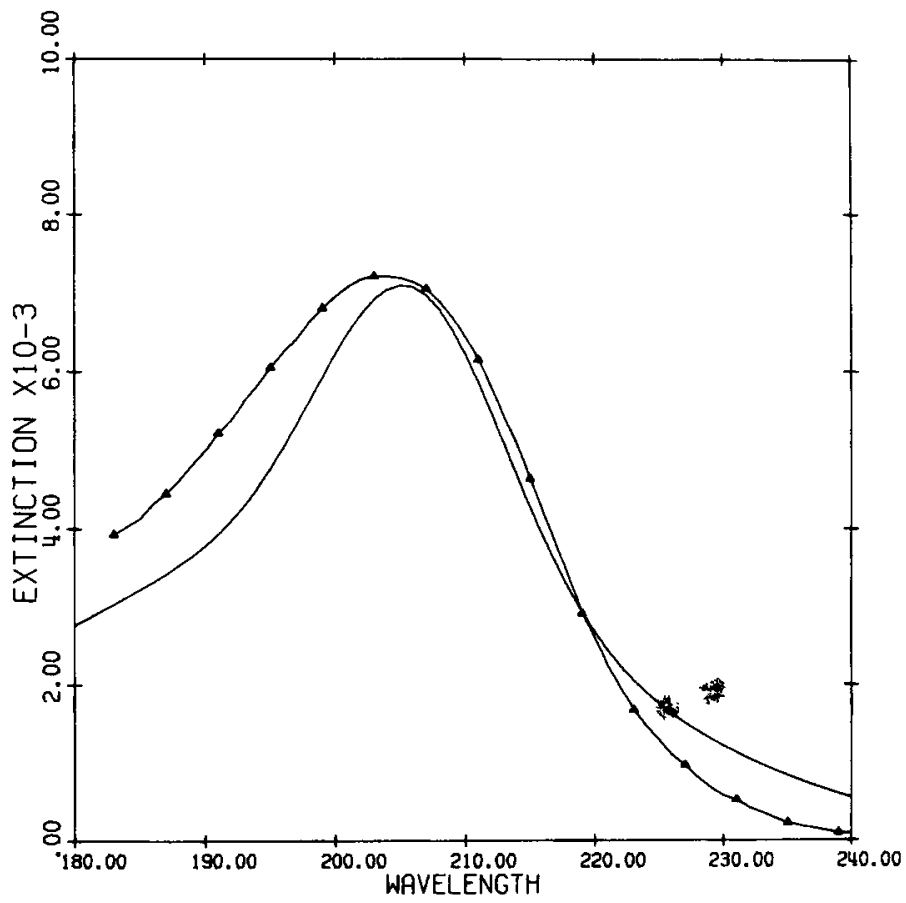

Fig. 5. Calculated (-) and observed ( $\Delta$ ) absorption spectra of polyproline II.

which amounts to $\mathbf{- 0 . 0 8 0}$ for the $\alpha$-helix.) Thus, the far uv transitions, while not dominating, make non-negligible contributions to the CD spectrum of the $\alpha$-helix. These are somewhat different in the $\pi \pi^{*}$ and $n \pi^{*}$ regions, the proportion bcing expected to depend on the conformation.

$\beta$ Structure. The calculated CD spectrum of a single chain in the $\beta$ conformation is shown in Figure 7, and some values of the rotational strengths are given in Table II. It is seen that pol- $R_{\pi \pi^{*}}$ is expected to be very small in this casc, whereas pol- $R_{n \pi^{*}}$ is predicted to contribute about $20 \%$ of the total $n \pi^{*}$ ellipticity. There are no prominent features predicted in the far uv, although this as well as the above conclusions might be modified somewhat as a result of a calculation based on a hydrogen-bonded sheet structure rather than a single chain.

Polyproline I. The calculated CD spectrum of PPI is shown in Figure 8, and some values of the rotational strengths are given in Table II. The calculated spectrum exhibits a small long-wavelength negative band, at about $235 \mathrm{~nm}$, which is not reproduced by previous calculations. ${ }^{1-3}$ This is a consequence of the incorporation of vibronic coupling, ${ }^{30}$ which places the $n \pi^{*}$ transition at $230 \mathrm{~nm}$ in the imides. The two strong CD bands are well reproduced in position, although less well so in their relative intensities.

Polyproline II. The calculated CD spectrum of PPII is shown in Figure 9 , and rotational strengths are given in Table II. It will be seen that good agreement is obtained in the $190-250-\mathrm{nm}$ region. The pol- $R_{\pi \pi *}$ contribution 


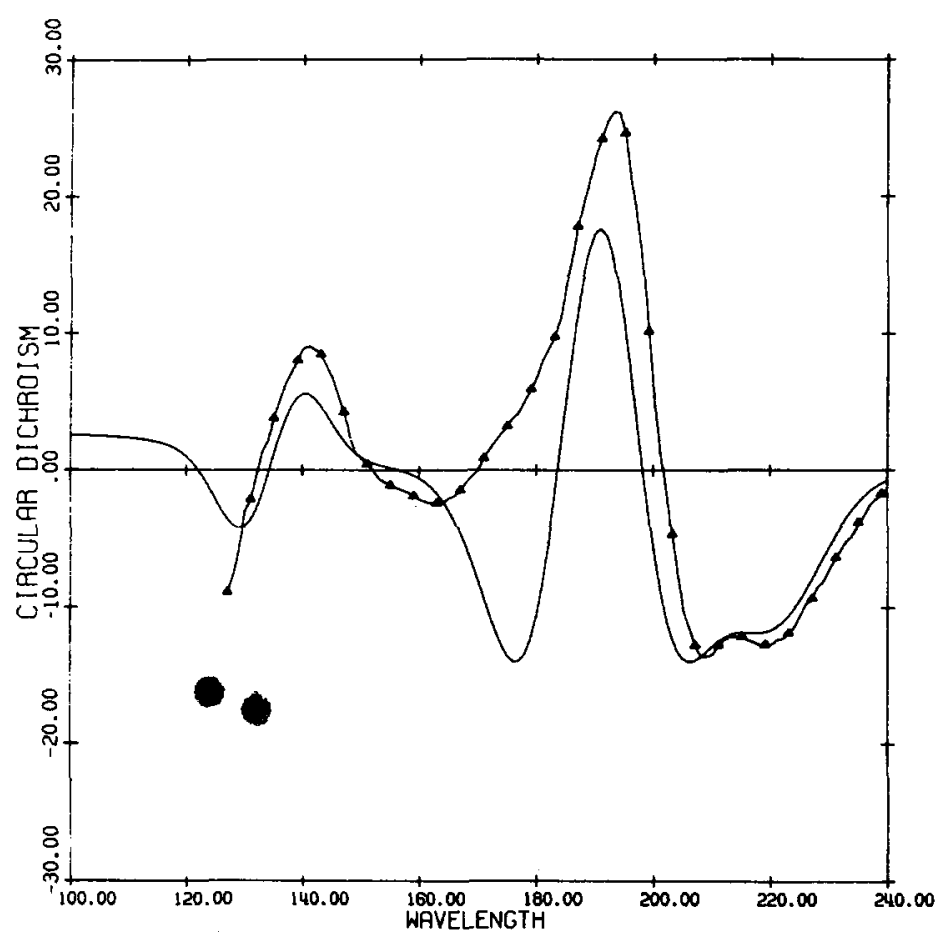

Fig. 6. Calculated (-) and observed ( $\triangle$ ) CD spectra of the $\alpha$-helix.

constitutes about $83 \%$ of the total observed ellipticity in this region. (The exc- $R_{\pi \pi^{*}}$ contribution is calculated to be small. This results from the fact that the positive and negative components are calculated to be large and close together: with bandwidthṣ of $30 \mathrm{~nm}$, they sum to a small net ellipticity.) The pol- $R_{n \pi *}$ intensity is calculated to be larger in magnitude than the total $R_{n \pi *}$ since its negative contribution is in part compensated by a positive exc- $R_{n_{\pi}}$ contribution. It should be noted that in our calculation the small positive band observed near $228 \mathrm{~nm}$ arises not from an $n \pi^{*}$ transition but from the overlap of the positive component of the $\pi \pi^{*}$ transition.

There are several features of the calculated PPII spectrum that are important. First, satisfactory agreement is obtained with the observed spectrum by using the appropriate conformation. ${ }^{15}$ Previous calculations achieved reasonable qualitative agreement only by invoking unacceptable conformations. $^{2-4}$ Second, the observed nonconservative spectrum in the 190-240-nm region is well reproduced. Earlier calculations ${ }^{2-4}$ predicted conservative spectra in this region. Third, our calculation predicts that the spectrum remains negative down to about $150 \mathrm{~nm}$. This is in good general agreement with recent observations, ${ }^{31}$ and must be considered a significant prediction of the present theory. The CD spectrum of PPII thus provides important evidence of the need to incorporate far uv transi- 


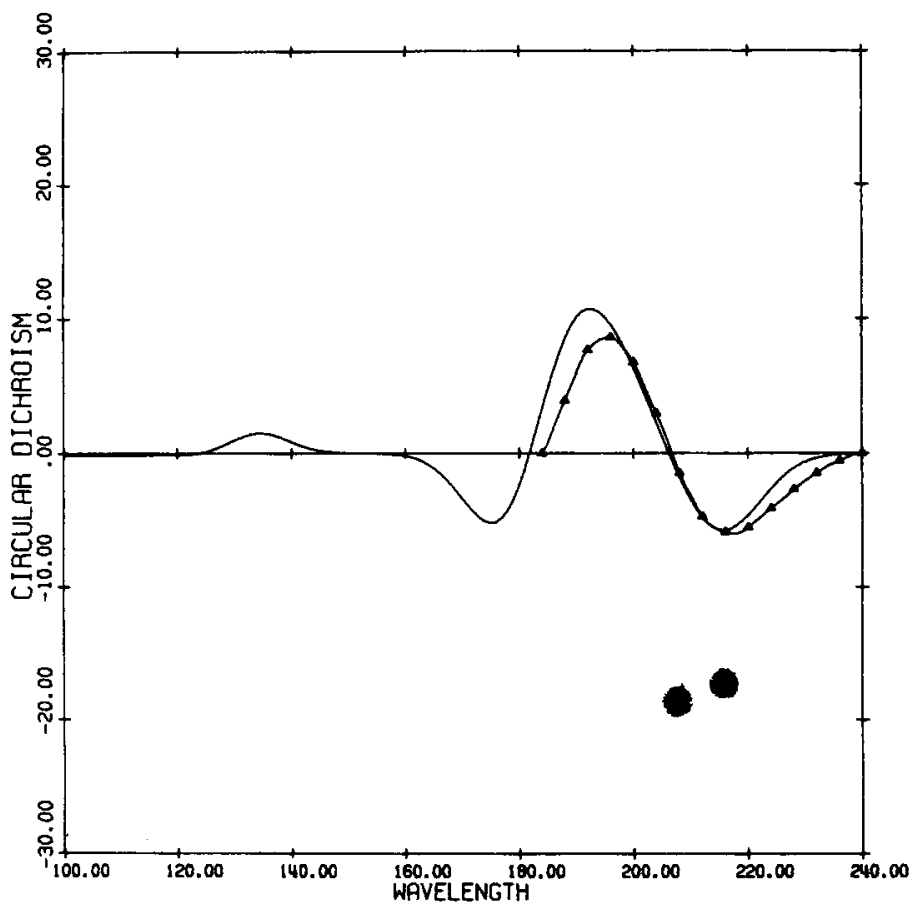

Fig. 7. Calculated $(-$ and observed $(\Delta) \mathrm{CD}$ spectra of the $\beta$ structure.

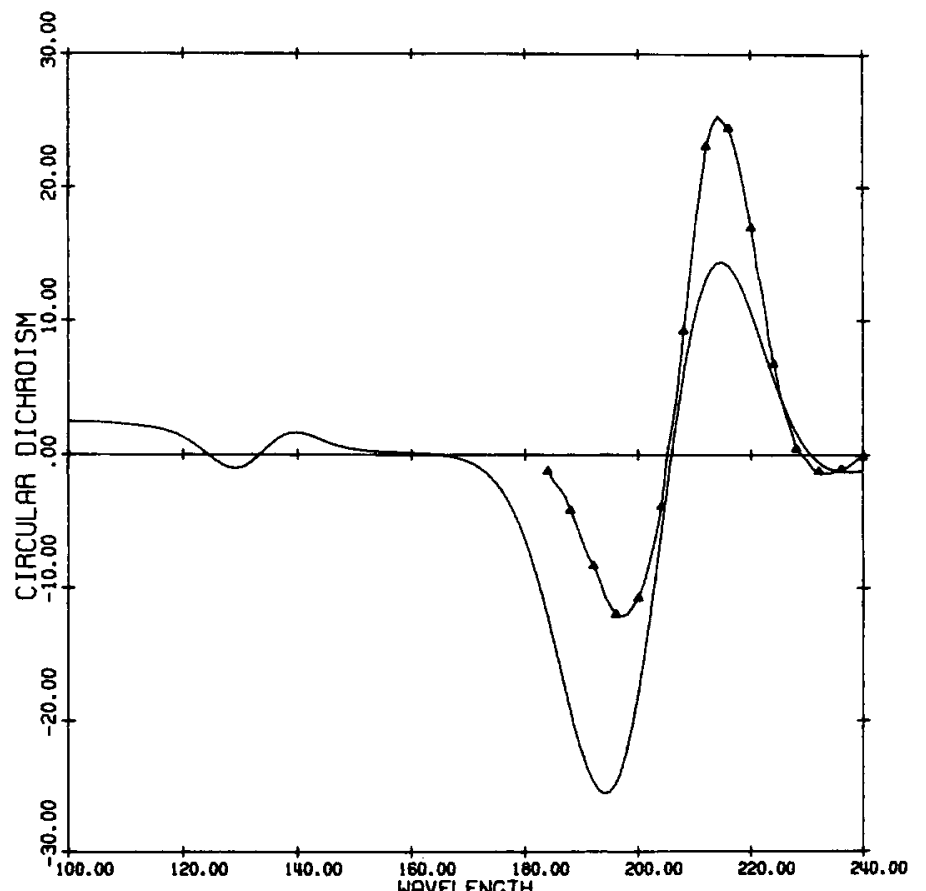

Fig. 8. Calculated ( $\longrightarrow$ ) and observed $(\Delta)$ CD spectra of polyproline I. 


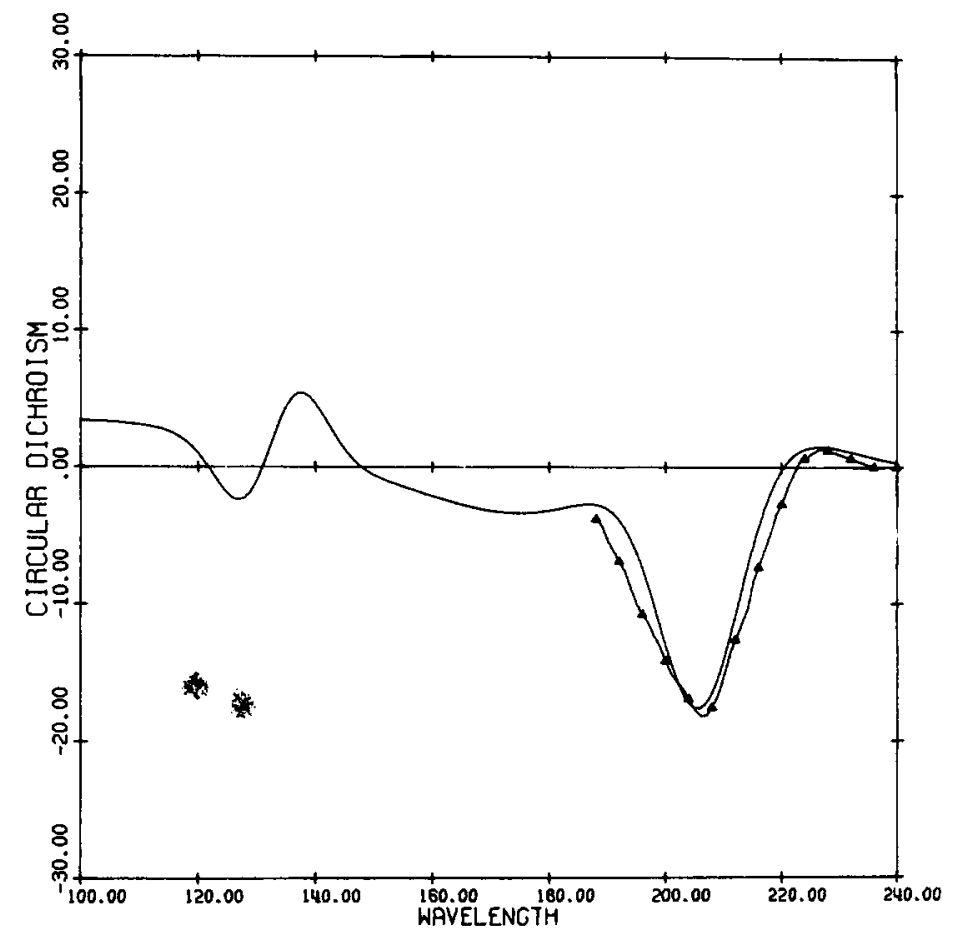

Fig. 9. Calculated ( $\longrightarrow$ ) and observed $(\Delta)$ CD spectra of polyproline II.

tions in the theoretical development. Our results support the general adequacy of the polarizability approximation in accounting for the main effect of these transitions, and provide, through the unique features of the PPII CD spectrum, a relatively reliable way of estimating the contributions made by these transitions.

It should be noted that our explanation of the small positive CD band near $228 \mathrm{~nm}$ is consistent with experimental observations of the loss of this band in $\mathrm{CaCl}_{2}$ solutions. ${ }^{32}$ The interaction of $\mathrm{CaCl}_{2}$ with the PPII chain is believed to lead to a disordering of the helical structure. ${ }^{32}$ This would be expected to lead to a relative decrease in the specific PPII exc- $R_{\pi \pi *}$ contribution to the spectrum, resulting in a loss of its overlap contribution at 228 nm.

Unordered Chains. A final word is in order concerning the expected effect of far uv transitions on the CD spectra of a maximally unordered system of polypeptide chains. We had shown previously ${ }^{10}$ that an exciton calculation predicts a conservative spectrum in the $180-250-\mathrm{nm}$ region, with a long-wavelength negative band. This is in general agreement with experimental observations, ${ }^{33,34}$ particularly in that a crossover to positive $\mathrm{CD}$ is seen at short wavelengths. ${ }^{34}$ We have examined the effects of including far uv transitions, in the polarizability approximation, on these 
results, and find the effect to be negligible. The basic reason seems to be that pol- $R_{\pi \pi *}$ and pol- $R_{n \pi *}$ can be positive or negative as a function of chain conformation, and these contributions cancel each other in a distribution of conformations represented by a maximally unordered chain. The previous calculations ${ }^{10}$ should therefore remain essentially valid for unordered polypeptides.

\section{CONCLUSIONS}

We have shown that, whereas exciton theory alone cannot account for the observed PPII CD spectrum for reasonable chain conformations, the inclusion of far uv transitions leads to good agreement with the observed spectrum. These transitions can be accounted for satisfactorily by a polarizability approximation. The calculation not only reproduces the observed nonconservative spectrum in the $180-250-\mathrm{nm}$ region, but predicts that the CD remains negative into the far uv. This was confirmed subsequently by experimental observation. ${ }^{31}$ The PPII spectrum thus constitutes an important system for providing an estimate of the contribution of far uv transitions in the CD spectra of polypeptide chains.

\section{APPENDIX}

The following equations give the polarizability corrections to the energy, dipole strength, and rotational strength. They are based on, and derived from, the equations given in Refs. 5 and 25.

\section{Energy}

The shift in the center of a transition, or the second order correction to the energy, is given by

$$
\begin{aligned}
E_{2}= & \frac{2}{N} \sum_{i=1}^{N} \sum_{j \neq i}^{N} \sum_{k \neq i}^{N} \frac{V_{i 0 a ; j 00} V_{i 0 a ; k 00}}{h \nu_{a}} \\
& -\frac{2}{N} \sum_{i=1}^{N} \sum_{j \neq i} \sum_{b \neq a} \frac{V_{i 0 a ; j 0 b} V_{i 0 a ; j 0 b}}{h\left(\nu_{b}-\nu_{a}\right)}
\end{aligned}
$$

where $N$ is the number of monomers, $a$ would be the $\pi \pi^{*}$ transition, and the sum is over all other groups and transitions in the helix.

\section{Dipole Strength}

The first order correction to the dipole strength, $H_{1}$, gives the total change in the area beneath the absorption curve (extinction coefficient versus wavelength). If there is an increase, the helix is said to be hy perchromic relative to the monomer. Only the $\alpha$-helix was calculated to be hypochromic.

$$
\begin{gathered}
H_{1}=-4 \sum_{j \neq i} \sum_{b} \mu_{i 0 a} \cdot T_{i j} \cdot \boldsymbol{\mu}_{j 0 b \mu_{j b b} \nu_{b}} \cdot \frac{\boldsymbol{\mu}_{i 0 a}}{h\left(\nu_{b}^{2}-\nu_{a}^{2}\right)} \\
T_{i j}=\left(1-\frac{\mathbf{R}_{i j} \mathbf{R}_{i j}}{\left|R_{i j}\right|^{2}}\right) \frac{1}{\left|R_{i j}\right|^{3}} .
\end{gathered}
$$


This term is further divided into a sum over transitions between 100 and $180 \mathrm{~nm}$ and transitions below $100 \mathrm{~nm}$. The latter are approximated by the polarizability ellipsoids.

$$
\begin{aligned}
H_{1}= & H_{1 b}+H_{1 w} \\
H_{1 w}= & \left\{-2 \sum_{j \neq i} \mu_{i 0 a} \cdot T_{i j} \cdot \hat{e}_{3} \hat{e}_{3} \cdot \mu_{i 0 a}\left(\alpha_{3 b}-\alpha_{11}\right)_{j}\right. \\
& \left.-2 \sum_{j \neq i} \mu_{i 0 a} \cdot T_{i j} \cdot \mu_{i 0 a}\left(\alpha_{11}\right)_{j}\right\} \frac{\nu_{0}^{2}}{\nu_{0}^{2}-\nu^{2}}
\end{aligned}
$$

In our case $H_{1 b}$ comes from the $\pi_{2} \pi^{*}$ transition; $\mu_{i 0 a}$ is the electric dipole moment of the $\pi \pi^{*}$ transition; $\hat{e}_{3}, \alpha_{33}, \alpha_{11}$ are the direction and magnitudes of the polarizability as given in Ref. $5 ; \nu_{0}=1 / \lambda_{0}$ where $\lambda_{0}=100 \mathrm{~nm}$ and $\nu_{a}=1 / \lambda_{\pi \pi^{*}}$ where $\lambda_{\pi \pi^{*}}=190 \mathrm{~nm}$ for the $\alpha$-helix.

\section{Rotational Strength}

The first order correction to the rotational strength is the net change, called pol- $R_{\pi \pi *}$ and pol- $R_{n \pi} *$ in the text, due to the contribution of the far uv transitions to the $\pi \pi^{*}$ and to the $n \pi^{*}$ transitions. The energy at which this rotational strength is added in is taken as a parameter, and a good fit seems to imply the $\pi \pi^{*}$ contribution adds at 204 $\mathrm{nm}$ in the amides and at $206 \mathrm{~nm}$ in the imides. The $n \pi^{*}$ contribution is added at the $n \pi^{*}$ wavelength. The band shape is achieved with a Gaussian band shape and the bandwidth is also a parameter.

$$
\begin{aligned}
\operatorname{pol}-R_{\pi \pi^{*}}= & \frac{1}{3}\left(R_{33}+R_{22}+R_{11}\right) \\
R_{33}= & -\sum_{i=1}^{N} 3 \pi \frac{\nu_{a} \nu_{0}^{2}}{\nu_{0}^{2}-\nu_{a}^{2}} \sum_{j \neq i} \frac{\left(R_{3 j}-R_{3 i}\right)}{c} \mu_{i 0 a} \cdot T_{i j} \\
& \cdot\left[\hat{e}_{3} \hat{e}_{3}\left(\alpha_{33}-\alpha_{11}\right)_{j}+\alpha_{11} 1\right] \cdot\left[i \mu_{y i 0 a}-j \mu_{z i 0 a}\right]
\end{aligned}
$$

where $\mathbf{R}_{j}$ is the position vector of ellipsoid (group) $j$ and $R_{3 j}$ is the $z$ component and the terms for $R_{22}$ and $R_{11}$ are similar.

$$
\begin{aligned}
\text { pol- } R_{n \pi *} & =\operatorname{Im}\left\{\frac { \nu _ { 0 } { } ^ { 2 } } { \nu _ { 0 } ^ { 2 } - \nu _ { a } ^ { 2 } } \sum _ { i = 1 } ^ { N } \sum _ { i } \frac { q _ { i t 0 a } } { | R _ { i t 0 a ; j } | ^ { 3 } } \left[\left(\alpha_{11}\right)_{j} \mathbf{R}_{i t 0 a ; j} \cdot \mathbf{m}_{i a 0}\right.\right. \\
& \left.\left.+\left(\alpha_{33}-\alpha_{11}\right)_{j}\left(\mathbf{R}_{i t 0 a ; j} \cdot \hat{e}_{3 j}\right)\left(\hat{e}_{3 j} \cdot \mathbf{m}_{i a 0}\right)\right]\right\}
\end{aligned}
$$

Here $a$ refers to the $n \pi^{*}$ energy and positions; $q, R$ are the $n \pi^{*}$ charge and monopole position; $\mathbf{R}_{i t 0 a ; j}=\mathbf{R}_{j}-\mathbf{R}_{i t 0 a}$ is the vector to the polarizability ellipsoid.

To these terms are added the corrections due to the $\pi_{2} \pi^{*}$ transition. To pol- $R_{\pi \pi *}$ is added $R_{\pi \pi}$ and to pol- $R_{n \pi}$ is added $R_{n \pi *}$ where $R_{\pi \pi} *$ and $R_{n \pi} *$ are given by the following equations:

$$
\begin{aligned}
R_{n \pi *}= & -\sum_{i=1}^{N}\left\{\sum_{j \neq i} \sum_{b \neq a} \operatorname{Im} V_{i a b ; j 00} \frac{\mu_{i 0 b} \cdot \mathbf{m}_{i a 0}}{h\left(\nu_{b}-\nu_{a}\right)}\right. \\
+ & \sum_{j \neq i} \operatorname{Im} V_{i 0 a ; j 00} \frac{\left(\mu_{i a b} \cdot-\mu_{i 00}\right) \cdot \mathbf{m}_{i a 0}}{h \nu_{a}} \\
R_{\pi \pi *}=- & \sum_{i=1}^{N}\left\{\frac{2 \pi}{c} \sum_{j \neq i} \sum_{b \neq a} V_{i 0 a ; j 0 b} \frac{\nu_{a} \nu_{b}\left(\mathbf{R}_{j}-\mathbf{R}_{i}\right) \cdot \mu_{j 0 b} \times \mu_{i 0 a}}{h\left(\nu_{b}^{2}-\nu_{a}^{2}\right)}\right\} .
\end{aligned}
$$


In these equations $a$ refers to the $\pi^{*}$ state, $\mathbf{m}_{00}$ is the $n \pi^{*}$ magnetic dipole moment, $\mu_{0 b}$ is the $\pi_{2} \pi^{*}$ electric dipole moment, and the $V_{i \theta a ; j o b}$ matrix elements are evaluated by a monopole approximation. These terms are derived in Refs. 5 and 25.

This research was supported by National Science Foundation Grant GB-15682.

\section{References}

1. Pysh, E. S. (1967) J. Mol. Biol. 23, 587-599.

2. Pysh, E. S. (1970) J. Chem. Phys. 52, 4723-4733.

3. Madison, V. \& Schellman, J. (1972) Biopolymers 11, 1041-1076.

4. Tterlikkis, L., Loxom, F. M. \& Rhodes, W. (1973) Biopolymers 12, 675-684.

5. Woody, R. W. \& Tinoco, I. (1967) J. Chem. Phys. 46, 4927-4945.

6. Woody, R. W. (1968) J. Chem. Phys. 49, 4797-4806.

7. Pysh, E. S. (1966) Proc. Nat. Acad. Sci. 56, 825-832.

8. Rosenheck, K. \& Sommer, B. (1967) J. Chem. Phys. 46, 532-536.

9. Woody, R. W. (1969) Biopolymers 8, 669-683.

10. Ronish, E. W. \& Krimm, S. (1972) Biopolymers 11, 1919-1928.

11. DeSantis, P., Giglio, E., Liquori, A. M. \& Ripamonti, A. (1965) Nature 206, 456458.

12. Schimmel, P. R. \& Flory, P. J. (1967) Proc. Nat. Acad. Sci. 58, 52-59.

13. Ramachandran, G. N., Lakshminarayanan, A. V., Balasubramanian, R. \& Tegoni, G. (1970) Biochim. Biophys. Acta 221, 165-181.

14. Krimm, S. \& Venkatachalam, C. M. (1971) Proc. Nat. Acad. Sci. 68, 2468-2471.

15. Venkatachalam, C. M., Price, B. J. \& Krimm, S., (1974) Macromolecules, 7, 212220 .

16. Rosenheck, K., Miller, H., \& Zakaria, A. (1969) Biopolymers 7, 614-618.

17. Mandel, R. \& Holzwarth, G. (1973) Biopolymers 12, 655-674.

18. Bovey, F. A. \& Hood, F. P. (1967) Biopolymers 5, 325-326.

19. Tiffany, M. L. \& Krimm, S. (1968) Biopolymers 6, 1767-1770.

20. Okabayashi, H., Isemura, T. \& Sakakibara, S. (1968) Biopolymers 6, 323-330.

21. Holzwarth, G. \& Doty, P. (1965) J. Amer. Chem. Soc. 87, 218-228.

22. Okabay ashi, H. \& Isemura, T. (1970) Bull. Chem. Soc. Japan 43, 20-23.

23. Moffitt, W. (1956) Proc. Nat. Acad. Sci. 42, 736-746.

24. Goodman, M., Listowsky, I., Masuda, Y. \& Boardman, F. (1963) Biopolymers 1, $33-42$.

25. Tinoco, I. (1962) Advan. Chem. Phys. 4, 113-160.

26. McMillan, C. R., Rippon, W. B. \& Walton, A. G. (1973) Biopolymers 12, 589-597.

27. Bayley, P. M., Nielsen, E. B. \& Schellman, J. A. (1969) J. Phys. Chem. 73, 228243.

28. Nielsen, E. B. \& Schellman, J. A. (1967) J. Phys. Chem. 71, 2297-2304.

29. Young, M. A. \& Pysh, E. S. (1973) Macromolecules 6, 790-791.

30. Lopata, E. S. \& Krimm, S., in preparation.

31. Pysh, E. S. private communication.

32. Tiffany, M. L. \& Krimm, S. (1968) Biopolymers 6, 1767-1770.

33. Tiffany, M. L. (1970) Ph.D. thesis, University of Michigan.

34. Tiffany, M. L. \& Krimm, S. (1972) Biopolymers 11, 2309-2316.

Received April 8, 1974 